\title{
Monetary incentives improve performance, sometimes: speed and accuracy matter, and so might preparation
}

\author{
Kimberly S. Chiew* and Todd S. Braver \\ Department of Psychology, Washington University in St. Louis, St. Louis, MO, USA \\ ${ }^{*}$ Correspondence: kschiew@wustl.edu
}

\section{A commentary on}

Monetary incentives in speeded perceptual decision: effects of penalizing errors versus slow responses

by Dambacher, M., Hübner, R., and Schlösser, J. (2011). Front. Psychology 2:248. doi: 10.3389/fpsyg.2011.00248

It is intuitive to assume that monetary rewards will improve cognitive performance. However, empirical research has yielded mixed results (Bonner et al., 2000). A new study by Dambacher et al. (2011) seeks to clarify an important factor - payoff schemes - in mediating the relationship between incentives and cognitive performance. Specifically, this paper presents a series of three experiments examining the effect of monetary versus symbolic incentives on performance in the Erikson flanker task (Eriksen and Eriksen, 1974). Fast, accurate performance was rewarded in all payoff schemes, while penalties for errors and slow responses were independently manipulated to examine the effects of emphasizing speed versus accuracy on performance.

Dambacher et al. (2011) highlight three main findings in their data: (1) performance improves under monetary incentive more when slow responses are punished than when they are not; (2) improvement is not observed when punishment for errors is emphasized instead; and (3) performance still improves without penalties as long as fast, accurate performance is emphasized with reward. Dambacher et al. (2011) interpret these results as evidence that emphasizing speed optimizes performance, while emphasis of both speed and accuracy (e.g., in Experiment 2) fails to enhance performance because determining an optimal response strategy is more difficult under these competing emphases. Importantly, this finding contributes toward a mechanistic understanding of when monetary incentives improve cognitive performance and when they do not.
Here we highlight certain aspects of the present study that warrant follow-up and further investigation.

One issue we wish to discuss is the role of deadline manipulations. In all three experiments of the present study, deadlines of varying lengths are used (long, medium, and short). Speed-accuracy tradeoff functions (SATFs) under each of these deadlines revealed speed-accuracy shifts in all conditions across studies, with speed increasing and accuracy decreasing with shorter deadlines. An alternative approach to examining speed-accuracy functions is use of diffusion model analysis (Ratcliff and McKoon, 2008; Ratcliff and Rouder, 1998), which provides quantitative estimation of contributions to decision performance. The diffusion model potentially sheds light on how such contributions change under incentive: i.e., whether incentive changes non-decision factors, such as stimulus encoding and response execution; the decision threshold (merely trading accuracy for speed); or specifically enhancing the quality of accumulated information (drift rate) via increased attentional effort, increasing performance speed while maintaining accuracy. A previous paper from the same group (Hübner and Schlösser, 2010) uses predictions from the diffusion model framework to evaluate flanker performance under incentive. They concluded that performance reflected increased speed while maintaining accuracy, consistent with predictions of increased drift rate as a result of increased attentional effort. However, neither that paper nor the current study include diffusion model analyses of the presented experimental data. Recent work suggests that the diffusion model provides an excellent account of behavioral performance, as well as the effects of attentional manipulations, in the flanker task (White et al., 2011). Thus, utilization of a diffusion model approach might provide a convergent means of verifying claims by Hübner, Dambacher et al. (2011) regarding how and when incentives influence task performance.
A potential disadvantage of the multiple deadline design employed by Dambacher et al. (2011) is that it may increase task complexity, without providing clear predictions regarding how incentive-related changes in the flanker effect should vary as a function of deadline length. Such differences were observed in the present study (as well as in Hübner and Schlösser, 2010), but remain relatively unexplained. In particular, in Experiment 3, the flanker effect decreased under incentive at short and medium deadlines, but not under the long deadline. These observations were interpreted as evidence that incentive can enhance selective attention without requiring penalty, but why this enhancement would take place at short and medium deadlines and not at longer deadlines remains unclear. Prior work suggests that incentive-related reductions in the flanker effect are elusive (Seifert et al., 2006). One reason may be that incentives have effects that might impact either nondecision time or drift rate, but that this could interact with the time available for responding. In their prior paper Hübner and Schlösser (2010) suggest that a signature of an incentive-related effect on nondecisional processing would be enhanced performance specifically at short deadlines, but decreasing effects at longer deadlines. Indeed, in Experiment 3, performance was enhanced at short and medium but not long deadlines, which might suggest a nondecisional effect.

Emerging data from our laboratory is consistent with the idea that monetary incentives may have mixed effects on cognitive performance and attentional control (Chiew and Braver, 2010). We observed a speed-accuracy shift under reward in a similar flanker paradigm, but only found incentive-related reduction of the flanker effect when participants observed a cue predicting the presence/absence of conflict in the upcoming array first. This is consistent with the idea that incentive may enhance attentional control specifically under conditions 
in which preparatory processes can be easily engaged (Savine and Braver, 2010). Moreover, preliminary diffusion model analysis of our data suggests that drift rate improved under incentive only in the presence of these preparatory cues, while the speed-accuracy shift under reward in the absence of these cues was associated with a change in both response caution and nondecision time.

An additional concern regards the simultaneous presence of rewards and punishments in Experiment 1 and 2 of the present study. Theoretical accounts link reward and punishment with approach and avoidance tendencies, respectively, thought to be dissociable, hemispherically lateralized influences on behavior (Davidson et al., 1990; Gray, 1994) distinctly impacting cognitive processes (Savine et al., 2010). Incentivized cognitive performance under emphasis of speed or accuracy should be carefully examined under reward versus penalty alone to better characterize these distinct motivational influences. We applaud Dambacher et al. (2011) for their initial investigations of how incentives affect task performance and attentional engagement during the flanker task. The extension of their work in the directions specified here should help to clarify the specific factors that determine how, why, and under what conditions incentives enhance cognitive processing and associated control functions.

\section{REFERENCES}

Bonner, S. E., Hastie, R., Sprinkle, G. B., and Young, S M. (2000). A review of the effects of financial incentives on performance in laboratory tasks: implications for management account. J. Manag. Account. Res. 12, 19-64.

Chiew, K. S., and Braver, T .S. (2010). Selective attention and conflict processing under primary motivational incentives: evidence from the flanker task. Poster Presented at the Annual Meeting of the Cognitive Neuroscience Society, Montreal, QC.

Dambacher, M., Hübner, R., and Schlösser, J. (2011). Monetary incentives in speeded perceptual decision: effects of penalizing errors versus slow responses. Front. Psychol. 2:248. doi: 10.3389/fpsyg.2011.00248

Davidson, R. J., Ekman, P., Saron, C. D., Senulis, J. A., and Friesen, W. V. (1990). Approach-withdrawal and cerebral asymmetry: emotional expression and brain physiology. I. J. Pers. Soc. Psychol. 58, 330-341.

Eriksen, B. A., and Eriksen, C. W. (1974). Effects of noise letters upon the identification of a target letter in a nonsearch task. Atten. Percept. Psychophys. $16,143-149$.

Gray, J. A. (1994). "Personality dimensions and emotion systems," in The Nature of Emotion, eds P. Ekman and R. J. Davidson (New York: Oxford University Press), 329-331.

Hübner, R., and Schlösser, J. (2010). Monetary reward increases attentional effort in the flanker task. Psychon. Bull. Rev. 17, 821-826.
Ratcliff, R., and McKoon, G. (2008). The diffusion decision model: theory and data for two-choice decision tasks. Neural Comput. 20, 873-922.

Ratcliff, R., and Rouder, J. N. (1998). Modeling response times for two-choice decisions. Psychol. Sci. 9, 347-356.

Savine, A. C., Beck, S. M., Edwards, B. G., Chiew, K. S., and Braver, T.S. (2010). Enhancement of cognitive control by approach and avoidance motivational states. Cogn. Emot. 24, 338-356.

Savine, A. C., and Braver, T. S. (2010). Motivated cognitive control: reward incentives modulate preparatory neural activity during task-switching. J. Neurosci. 30, 10294-10305.

Seifert, J., Naumann, E., Hewig, J., Hagemann, D., and Bartussek, D. (2006). Motivated executive attention - incentives and the noise-compatibility effect. Biol. Psychol. 71, 80-89.

White, C. N., Ratcliff, R., and Starns, J. J. (2011). Diffusion models of the flanker task: discrete versus gradual attentional selection. Cogn. Psychol. 63, 210-238.

Received: 18 October 2011; accepted: 21 October 2011; published online: 09 November 2011.

Citation: Chiew KS and Braver TS (2011) Monetary incentives improve performance, sometimes: speed and accuracy matter, and so might preparation. Front. Psychology 2:325. doi: 10.3389/fpsyg.2011.00325

This article was submitted to Frontiers in Cognition, a specialty of Frontiers in Psychology.

Copyright $(2011$ Chiew and Braver. This is an open-access article subject to a non-exclusive license between the authors and Frontiers Media SA, which permits use, distribution and reproduction in other forums, provided the original authors and source are credited and other Frontiers conditions are complied with. 